\title{
Introduction: Advances in brain-machine interfaces
}

\author{
Parag G. Patil, M.D., Ph.D. \\ Departments of Neurosurgery and Biomedical Engineering, University of Michigan, Ann Arbor, Michigan
}

$\mathrm{S}$ ince the seminal discovery by Gustav Fritsch and Eduard Hitzig in $1870^{2}$ that electrical stimulation of the canine cortex produces motor activity in the limbs, there has been considerable scientific interest in the interface of the brain with electrical devices. Neurosurgeons have collaborated with other neuroscientists throughout the development of the field. As early as 1901, Harvey Cushing spent several months in England working with neurophysiologist Sir Charles Sherrington, distinguishing motor and sensory cortex with respect to the central sulcus through electrical stimulation. Research related to brain-machine interfaces has always been translational. In 1909, after returning to Johns Hopkins University, Cushing performed the first sensory stimulation in the awake human patient. ${ }^{1}$

Advancement in the field awaited further understanding of cortical and subcortical functional organization, the development of noninvasive brain imaging modalities, and the advent of high-speed computing. Today the field of brain-machine interfaces encompasses cellular and systems neurobiology, materials science, electrical engineering, computer science and information theory, robotics, imaging, optics, and other engineering, scientific, and medical disciplines. Functional neurosurgical procedures utilizing brain-machine interfaces are performed daily in the form of deep brain stimulation (DBS) surgery for movement disorders, cortical mapping during eloquent cortex tumor resection, motor cortex stimulation for pain, and the placement of subdural grid electrodes for seizure localization. Notable recent advances in the field include DBS for the treatment of depression ${ }^{4,6}$ and obsessive-compulsive disorder, ${ }^{5}$ a clinical trial to study responsive cortical stimulation for the treatment of epilepsy, ${ }^{8}$ and the report of a successful human brain-machine interface motor neuroprosthetic device. ${ }^{3}$

In this issue of Neurosurgical Focus, the contributing authors present insightful reviews of the brain-machine interface field as well as their original scientific work. In the first of 5 reviews, Awan and colleagues present current and future perspectives of DBS therapy. Hu and colleagues then review the neuroanatomy of memory and explore the potential of DBS to modulate memory formation and recall. Leuthardt and colleagues present the current state of the field for the conversion of brain signals into machine commands for neuroprosthetic ap- plications. Ryu and Shenoy examine the unmet needs of current brain-machine interface approaches in terms of system durability, performance, and surgical risk. Shifting attention to the potential of brain-machine interfaces for sensory neuroprosthetic applications, Pezaris and Eskandar review the field of visual prosthetics and the potential for thalamic stimulation to mimic vision. Finally, Denning and colleagues thoughtfully explore the potential information security implications of motor and sensory neuroprosthetic devices.

Advances in the brain-machine interface field are represented in 6 reports of original work. Rohatgi and colleagues report on the in vivo performance of a microelectrode neural probe with integrated drug delivery. Two subsequent papers by Kellis and Leuthardt and their colleagues report on the application of high-density cortical surface arrays for neuroprosthetic applications. Papers by Reddy and colleagues and Scherer and associates examine the ability of cortical surface recordings to differentiate movement direction and limb laterality. Finally, Blakely and colleagues report a clinical example of a highly stable algorithm for cursor control.

Significant challenges remain to the future advancement of brain-machine interfaces. Signal detection, information extraction, actuator development, issues of feedback, and neural adaptation each pose significant scientific and technological hurdles for scientists in the field. ${ }^{7}$ Even so, there is significant interest on the part of the National Institutes of Health and other funding agencies to support brain-machine interface research and to catalyze interdisciplinary research. The final paper in the issue is an editorial overview by Dr. Pancrazio of the National Institute of Neurological Disorders and Stroke, describing the opportunities and programs currently available to support brain-machine interface projects.

I thank the individual authors, the reviewers, Neurosurgical Focus Editor Dr. Martin H. Weiss, and Assistant to the Editor Margie A. Shreve for their efforts and contributions to this issue.

\section{References}

1. Cushing H: A note upon the faradic stimulation of the postcentral gyrus in conscious patients. Brain 32:44-53, 1909 
2. Fritsch G, Hitzig E: Ueber die elektrische Erregbarkeit des Grosshirns. Arch Anat Physiol 3:330-332, 1870

3. Hochberg LR, Serruya MD, Friehs GM, Mukand JA, Saleh M, Caplan AH, et al: Neuronal ensemble control of prosthetic devices by a human with tetraplegia. Nature 442:164-171, 2006

4. Lozano AM, Mayberg HS, Giacobbe P, Hamani C, Craddock RC, Kennedy SH: Subcallosal cingulate gyrus deep brain stimulation for treatment-resistant depression. Biol Psychiatry 64:461-467, 2008

5. Mallet L, Polosan M, Jaafari N, Baup N, Welter ML, Fontaine $\mathrm{D}$, et al: Subthalamic nucleus stimulation in severe obsessive- compulsive disorder. NEJM 359:2121-2134, 2008

6. Malone DA, Dougherty DD, Rezai AR, Carpenter LL, Friehs GM, Eskandar EM, et al: Deep brain stimulation of the ventral capsule/ventral striatum for treatment-resistant depression. Biol Psychiatry 65:267-275, 2009

7. Patil PG, Turner DA: The development of brain-machine interface neuroprosthetic devices. Neurotherapeutics 5:137146, 2008

8. Sun FT, Morrell MJ, Wharen RE Jr: Responsive cortical stimulation for the treatment of epilepsy. Neurotherapeutics 5:68-74, 2008 